\title{
Editorial
}

\section{Cranial orthosis therapy}

James Tait GoOdrich, M.D., Ph.D.

Division of Pediatric Neurosurgery, Leo Davidoff Department of Neurosurgery, Children's Hospital at Montefiore, Albert Einstein College of Medicine, Bronx, New York

I read with interest this follow-up study by Dr. Jimenez and Dr. Barone on their surgical treatment of coronal synostosis. ${ }^{1}$ The study reviews a 16-year experience with 115 patients who were surgically treated with an endoscopy-assisted craniectomy supplemented with postoperative cranial orthosis therapy (helmet-assisted recontouring of the skull deformity). I have been familiar with this work since its beginning and have participated in a number of panel discussions with Dr. Jimenez at national and international meetings over the years. As a result I am intimately familiar with the work of this team and have looked forward to seeing what their outcomes are in the long term.

The authors nicely review the history of the strip craniectomy starting with ill-founded beginnings in the 1880s and 1890s by Lannelongue and Lane among a number of other not-so-insightful surgeons. Based on Rudolf Virchow's paper on cretinism and microcephaly, the concept of the suture as the cause of craniosynostosis led a number of nineteenth-century general surgeons to remove fused sutures to treat "idiocy." Thanks to a New York pediatrician by the name of Abraham Jacobi, who issued a scathing report detailing an enormously high mortality rate and poor results with this technique, suture craniectomy surgery came to a halt by the early 1900 s. With the work of Ingraham and Matson the strip craniectomy came back into focus for neurosurgeons in the 1940s. Because the earlier procedures had not accomplished the desired goals, these surgeons decided to add silicone wrappers to the craniectomy edges and apply metal clips, with the intended effect of restricting rapid bone regrowth. Others supplemented the technique by adding Zenker's solution to the dura among other things, only to find the morbidity rate to be excessively high. The strip technique, however, remained popular until well into the 1970s and 1980s, and was the technique I was trained to perform as a resident. With the work of Paul Tessier, Daniel Marchac, and Fernando Ortiz-Monestario among others, the cranial vault remodeling techniques, which are now widely used throughout the world, came to be adopted by both neurosurgeons and plastic surgeons. Cranial vault remodeling has remained popular, as the surgical teams performing these cranial vault remodeling procedures believe they are better able to achieve more favorable outcomes when compared with strip craniotomies. According to the detractors, the downside to cranial vault remodeling is the longer operating time, more blood loss, uneven surface contours, and greater hospital expense due to increased length of stay. In an effort to improve outcomes in strip craniectomy the authors added the orthotic helmet as an additional correction factor to prevent the relapse commonly observed in routine strip craniectomies; the question to ask is, have these technical changes improved this surgery, a thought that I will address later.

One of the questions in dealing with any of the patients with craniosynostosis is, where are the biological factors coming from that lead to these multifocal deformations? To those who advocate the strip craniectomy, the suture is clearly the primary focus and the secondary changes noted in the craniofacial skeleton (such as orbital dystopia, facial scoliosis, and others) are secondary to the closed suture. Then there are those craniofacial surgeons who believe that the scalp, skull (and suture), muscle, and surrounding envelope of soft tissue all form a complex functional matrix with strong and important interactions driven by the skull base and the soft tissue matrix surrounding it. Although many investigators have researched this concept, the leader was Dr. Melvin Moss, who in the 1950s through the 1990s published a series of complex anatomical papers in which he detailed his views of the "functional matrix theory." It is beyond the concept of a commentary to detail this work, but I will summarize his view with this quotation: "...the growth and development of the head, in both normal and pathological states, is primarily regulated by the development of the 'soft' nonskeletal parts, and cranial growth is a secondary phenomenon." ${ }^{2,3}$ It is on the basis of this principle that I, and other craniofacial surgeons, have had a long and difficult time understanding why a strip craniectomy would work in such a complex biological interaction. I have had this discussion many times with Dr. Jimenez, and we clearly have differences in our views, but having said that, I was most

This article contains some figures that are displayed in color online but in black-and-white in the print edition. 
intrigued to read this paper and see the modifications they offered and what they had done to achieve better results using a strip craniectomy with a 16-year follow-up.

The issues that I am most interested in deal with the resultant long-term head shape and, most importantly, with preventing the known high relapse rates that occur in strip craniectomy. Our craniofacial center was established in 1958 and is the oldest in the country, and from our registry of more than 6000 patients we have been able to follow these children closely. It has been our impression that there are 3 age periods in which a relapse can occur in a craniosynostosis repair: within the first year of surgical treatment, around school age (typically 5-8 years of age), and a third period in the teenage years. This information is not new, and realizing that these relapses occur led this surgical team to design additional molding treatments to prevent these undesirable relapses; in this study by Jimenez and Barone it was the use of orthotic molding helmets designed to align the desired growth pattern. When the endoscopy-assisted technique was introduced, helmets were only placed for 4 to 6 months to achieve the desired correction. Over the last several years, during panel discussions on the use of helmets, I have seen the length of helmet placement significantly increase. At a recent panel meeting in which helmets were discussed, it was apparent that helmets are now commonly in place for 15 to 18 months. When I asked why this length of time was required, the answer was that this was being done because patients were "relapsing" and redeveloping their original deformity. The next obvious question one might want to ask is, what was then done to correct those children who had relapsed? In our experience with positional plagiocephaly and helmets, the window of correction is under 1 year of age. An additional query is the cost as one of the arguments for this technique is cost reduction to the health care system. If one figures in the 2 to 4 additional helmets needed, how does that factor into the overall cost of care? In our system these helmets cost $\$ 3000$, so I see an additional $\$ 6000$ to $\$ 12,000$ cost that negates any hospital inpatient savings. In addition, keeping a child in a helmet for more than 1 year and up to 18 months seems cruel and inhumane.

Another thought that I keep stumbling over as I read these reports is what happens to the craniectomy defect over time. Over the years I had heard much discussion about the ability of the stripped bone to regenerate and thereby not leave the undesirable persistent bone defects that we commonly experienced in the 1980 s. In my practice I continue to see a routine stream of patients who seek consultations on how to deal with the defects that remain from strip craniectomy procedures. If one researches papers on cranioplasty repairs and looks closely at the patient base, there are clearly a significant number of patients who are being treated with "cranioplasties" due to large and persistent skull defects from strip craniectomies. It was interesting to note the authors make a comment in the paper that they have had to reduce the size of the craniectomies to reduce this problem, but it is not clear what was eventually done to those earlier patients who remained with persistent defects.

In these authors' hands the children have had very low morbidity and no deaths, for which they are to be commended. To me this study is a tour-de-force because the operation is performed on a child less than 3 months of age, the desired age at which to perform these strip procedures. As I review the criteria for surgery and young ages, the associated risks are, to my mind, enormous. Our craniofacial team has not routinely performed an elective craniofacial procedure in a child under 4 months of age in more than 25 years. We made this decision for a number of reasons, but the most important one to our team is the higher risk of disseminated intravascular coagulation in the immature hematological system, which in most children does not mature until approximately 4 months of age. The young infant $(<4$ months of age) is a very fragile ecosystem with a cardiac system that poses not insignificant risks, including persistent patent foramen ovale and patent ductus arteriosus among other findings, all of which can increase the risk of pulmonary embolism in these surgical procedures.

After having attended many craniofacial meetings around the world, in addition to my time spent as an educator in the European Society of Pediatric Neurosurgeons postgraduate courses, I am intrigued with how many surgeons are now discussing significantly different outcomes with higher morbidity and mortality, all associated with the risks discussed above. In reviewing other patient series using these techniques, it has become clear to me that at other institutions the operating times are longer, hospital stays are longer, and blood transfusion rates are higher, when compared to the results reported in this paper. More disturbing is the situation in which mortality and morbidity are discussed only subliminally. In the end, a number of surgeons have again discontinued strip craniectomy for the reasons discussed.

A disturbing additional factor is the widespread lack of follow-up of these children in neurosurgical practices. The routine course is to perform the craniectomy, followup with the child postoperatively for maybe a year, and then they are "lost" to follow-up. In a craniofacial practice the team is obligated to follow these children into the teenage years, to not only document outcome but also to document relapse or other adverse results. These authors report a 16-year follow-up course, which is excellent, but I would like to have learned of the teenagers and their outcomes: the oldest postoperative patient in the paper was followed for only 8 years postoperatively.

In reviewing strip craniectomies the palimpsestic history is one of ironies, in that it is an operation that keeps arising out of the domains of history. After a scathing diatribe by Abraham Jacobi the technique died out in the 1890s. The procedure was resurrected in the 1940s and modified with Silastic implants, and was popular into the early 1980 s, only to be replaced by the cranial vault remodeling techniques because surgeons were just not satisfied with the results. In my own personal minimifidian experience the strip craniectomy was not a good procedure, leaving me disappointed with the results, and I now have no interest in reviving this technique. Even with all the modifications to the strips over the years-whether they be extended barrel-staves, use of Silastic, or othersthe results have not been as good as would be expected 


\section{Editorial}

from reviewing the literature and attending meetings. But even more basic to the understanding of which surgical technique you will use in craniosynostosis can be reduced to a simple concept: do you believe the suture is the origin of the problem, or is it the surrounding matrix, that is, the functional matrix, that determines the growth of the skull and skeletal matrix? Dr. Derek Bruce made the comment a number of years ago at a national meeting, when he was asked "What is the suture for?" and he responded "... it is there to allow the child to come through the birth canal," and I could not agree more.

Despite much discussion over the years with Dr. Jimenez I have not been able to convince him of my views nor has he been able to convince me of his. I will continue to follow his work and look forward to seeing these children in their teens with nonstigmatized heads and no bone defects. I will also continue to follow the goals of Dr. Jimenez and Dr. Barone of reducing blood loss, operating times, and lengths of stay; hopefully, all surgeons are also doing this in the age of increasing outcome monitoring. Having said that, why is it necessary to keep a child in a helmet for 12 to 18 months when the surgical team can add just 1 to 1.5 hours to the procedure and have the immediate result in front of them? Humanum est errare. (http://thejns.org/doi/abs/10.3171/2013.1.PEDS12581)

\section{Disclosure}

The author reports no conflict of interest.

\section{References}

1. Jimenez DF, Barone CM: Early treatment of coronal synostosis with endoscopy-assisted craniectomy and postoperative cranial orthosis therapy: 16-year experience. Clinical article. J Neurosurg Pediatr [epub ahead of print June 28, 2013. DOI: 10.3171/2013.4.PEDS11191]

2. Moss ML: Functional cranial analysis and the functional matrix, in Wertz RT (ed): Proceedings of the Conference Patterns of Orofacial Growth and Development. Washington, DC: American Speech and Hearing Association, 1971, pp 5-19

3. Moss ML: The functional matrix hypothesis revisited. 1. The role of mechanotransduction. Am J Orthodon Dentofac Orthoped 112:8-11, 1997

\section{Response}

David F. Jimenez, M.D., ${ }^{1}$ and Constance M. Barone, M.D. ${ }^{2}$

Departments of ${ }^{1}$ Neurosurgery and ${ }^{2}$ Plastic Surgery, UT Health Science Center at San Antonio, Texas

Dr. Goodrich's commentary exemplifies the way that a classically trained neurological surgeon and avid historian continues to practice and live in the past. It is indeed human to err, but one must learn from the recurring, pesky poor results that intransigence often refuses to acknowledge. His commentary leads one to wonder if he is still a strong proponent of "functional lambdoid synostosis" theory and still operates on patients with positional plagiocephaly as he so often did in the past. He continues to be a strong believer and proponent of Moss' theory (which remains only a theory); we simply do not share his belief, and our clinical experience demonstrates why. We have shown in our study of multiple-suture nonsyndromic craniosynostosis that the reverse occurs: calvarial suture synostosis can lead to significant deformational changes of the skull base, which can be fully corrected with early suture release; ${ }^{1}$ such events cannot be explained by Moss' concepts (Fig. 1).

Dr. Goodrich's historical quotations regarding the use of Silastic strips or Zenker's solution are simply irrelevant to the current discussion. Of course it makes no sense that placing a plastic barrier at a bone's edge would stop ossification, as new bone does not originate at the edges, but does so at the level of the osteogenic dura. Moreover, we do not cauterize the dura, and handle it minimally; doing otherwise will destroy the osteogenic cells and lead to large calvarial defects. Over the years we have observed surgeons use bipolar electrocautery to manage dural oozing; perhaps that is why the many unnamed surgeons that he quotes report large osseous defects - in surgery, it is all about technique!

We fully agree that long-term follow-up is necessary to corroborate whether or not surgical corrections will last or relapse will develop. That is precisely why we waited 16 years to publish this work, and believe the results speak for themselves (Fig. 2).
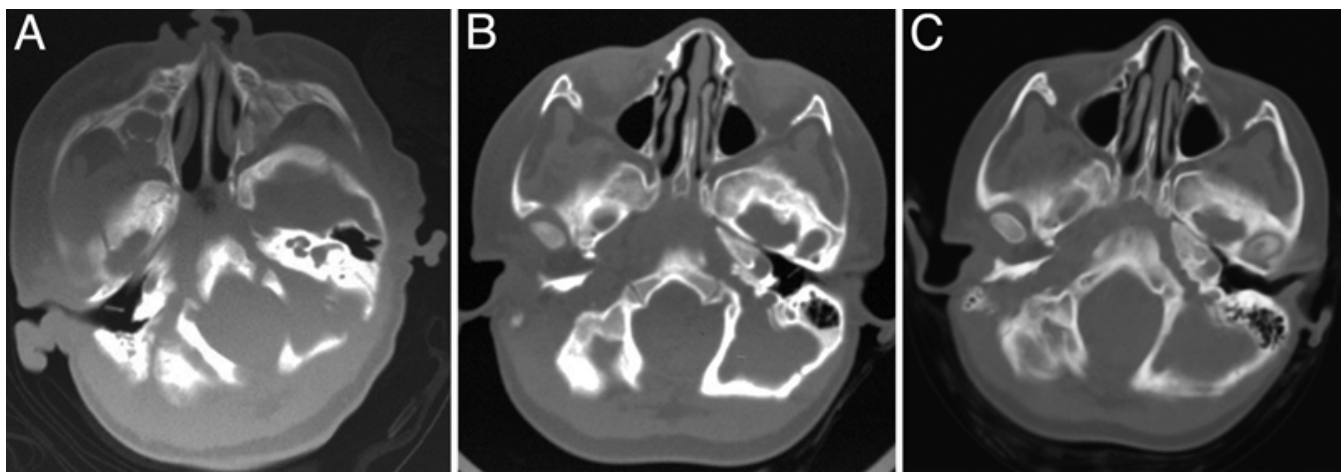

FıG. 1. Preoperative (A), 2-year postoperative (B), and 6-year postoperative (C) axial CT scans of an 8-week-old girl with left coronal, left lambdoid, and sagittal synostosis. The preoperative scan shows significant sagittal deviation at the skull base, with marked deviation of the foramen magnum from the midline $(A)$. The patient underwent suturectomies of the involved sutures. The 2-year scan shows progressive realignment of the foramen magnum and the midline sagittal plane (B), whereas the 6-year scan demonstrates complete realignment of the foramen magnum with the sagittal plane and coronal balance of the petrous bones (C). 

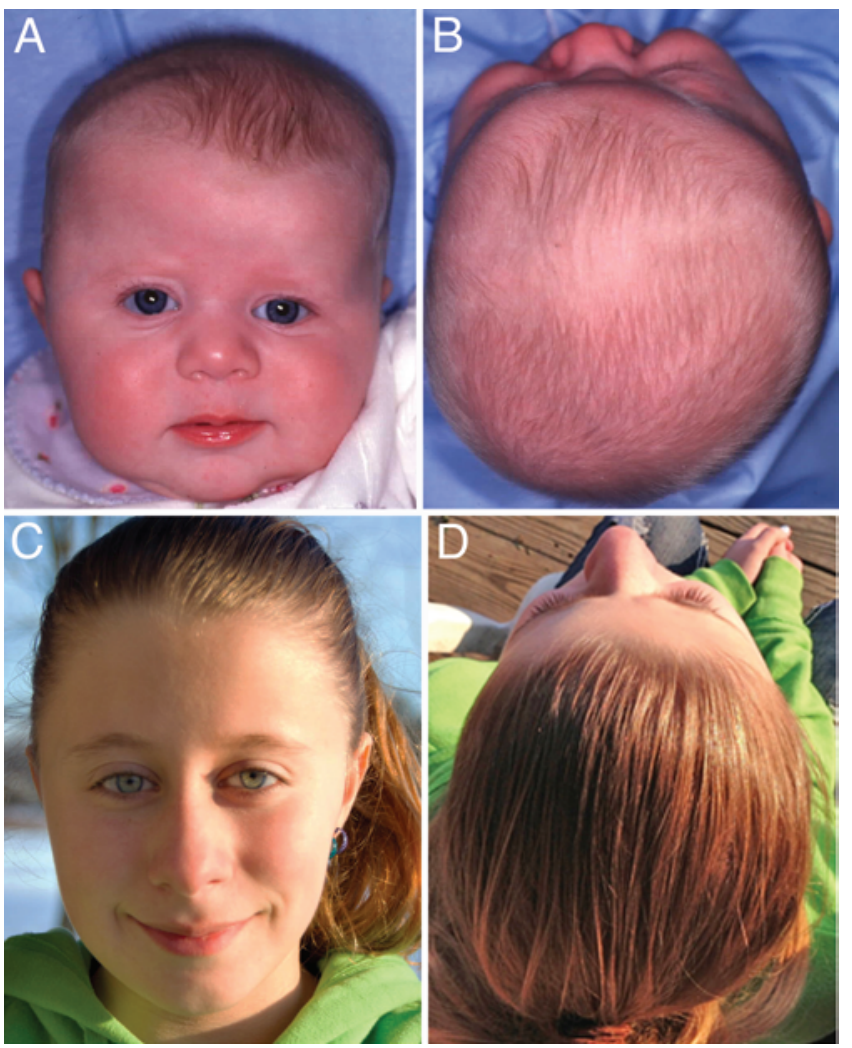

FIG. 2. Preoperative (A and B) and 12-year postoperative (C and D) photographs of an infant girl who presented with right coronal craniosynostosis. The preoperative photographs obtained when the girl was 12 weeks old show vertical dystopia, nasal deviation, and midsagittal imbalance of the craniofacial skeleton (A). The overhead view shows right frontal plagiocephaly (B). The postoperative anteroposterior image obtained 12 years after endoscopic craniectomy shows complete correction of vertical dystopia (C). The overhead view (D) also demonstrates correction of the forehead asymmetry. Photos provided by the parents of the child.

Dr. Goodrich is confused about our concepts regarding cranial orthotic therapy. From the very beginning, we began to use the orthoses for 10 to 12 months. We have indeed stated that there is a high tendency for relapse in patients with sagittal synostosis, which is the very reason why we argue for their long-term use. For sagittal synostoses our approach is as follows: Phase 1 (0-1 months) correction of scaphocephaly; Phase 2 (2-6 months), overcorrection with a goal of cephalic indices in the mid to high 80s; Phase 3 (6-12 months), maintenance of overcorrection and counteracting relapse forces. Ultimately, the cephalic indices of patients fall to the low 80s, thereby demonstrating excellent results. Coronal and metopic patients do not have the same tendency for relapse as those with sagittal synostoses. Our experience does not show relapse. Why would an orbit that has fully realigned along the horizontal axis suddenly elevate itself and become dystopic when the child is 2,3 , or 5 years old? This is nonsense. Because Dr. Goodrich does not use helmets, we wonder how many parents he has spoken to who corroborate that they have been cruel and inhumane with their children for placing them in an orthosis. In our experience with more than 500 patients and 1500 helmets, we have found no parents to corroborate this claim. The helmet becomes part of the child's routine just like socks, shirts, and pants do. We find it more cruel and inhumane to subject patients to secondary (and sometimes tertiary) "touch up" surgeries that are so often necessary using the traditional calvarial vault remodeling techniques.

We would like to address the concern for operating on babies younger than 3 months of age. Surgeries on newborns (such as those with myelomeningoceles, for example) have been performed for a long time without significant complications or deaths. Any competent surgical and anesthesia team can operate on newborns without significant concerns, yet performing a massively invasive calvarialvault remodeling procedure on a very young infant would be associated with significant morbidity and risk of death. That is precisely why we developed the minimally invasive endoscopic surgery that we report in our paper, and exactly why it is so well tolerated and there are minimal problems associated with it. We do not recall ever performing a complex myelomeningocele repair in less than 45 minutes, yet most of our coronal procedures are completed in less time.

Lastly, while it is interesting to read the lengthy commentary by Dr. Goodrich, he does not address a single result presented in the report. He has no comments on blood loss, transfusion rates, surgical time, length of stay, rates of vertical dystopia, or midsagittal deviation correction, to name a few. We have yet to see Dr. Goodrich publish his results on correction of vertical dystopia, nasal deviation, or sagittal imbalance in any of his papers on coronal synostosis. We encourage readers to carefully look at these outcomes and decide for themselves, and not simply base their conclusions on a few faulty historical facts.

\section{Reference}

1. Jimenez DF, Barone CM: Multiple-suture nonsyndromic craniosynostosis: early and effective management using endoscopic techniques. Clinical article. J Neurosurg Pediatr 5: 223-231, 2010

Please include this information when citing this paper: published online June 28, 2013; DOI: 10.3171/2013.1.PEDS12581. 